TRANSACTIONS OF THE

AMERICAN MATHEMATICAL SOCIETY

Volume 360, Number 11, November 2008, Pages 6113-6129

S 0002-9947(08)04499-1

Article electronically published on June 16, 2008

\title{
INDUCING PRIMITIVE IDEALS
}

\author{
SIEGFRIED ECHTERHOFF AND DANA P. WILLIAMS
}

\begin{abstract}
We study conditions on a $C^{*}$-dynamical system $(A, G, \alpha)$ under which induction of primitive ideals (resp. irreducible representations) from stabilizers for the action of $G$ on the primitive ideal space $\operatorname{Prim}(A)$ give primitive ideals (resp. irreducible representations) of the crossed product $A \rtimes_{\alpha} G$. The results build on earlier results of Sauvageot and others and will correct a (possibly overly optimistic) statement of the first author. In an appendix, the first author takes the opportunity to fill a gap in the proof of an earlier result.
\end{abstract}

\section{INTRODUCTION AND STATEMENT OF RESULTS}

In this paper we examine conditions on a $C^{*}$-dynamical system $(A, G, \alpha)$ so that induction of primitive ideals from stabilizers always leads to primitive ideals of the (full) crossed product $A \rtimes_{\alpha} G$. For any $C^{*}$-algebra $B$, we denote by $\mathcal{I}(B)$ the set of all closed two-sided ideals of $B$ equipped with Fell's topology (see [6, Chapter 1] for the definition). Recall (cf. [19, §3.3]) that there are continuous maps

$$
\operatorname{Ind}_{H}^{G}: \mathcal{I}\left(A \rtimes_{\alpha} H\right) \rightarrow \mathcal{I}\left(A \rtimes_{\alpha} G\right) \quad \text { and } \quad \operatorname{Res}: \mathcal{I}\left(A \rtimes_{\alpha} G\right) \rightarrow \mathcal{I}(A)
$$

characterized by

$$
\operatorname{Ind}_{H}^{G}(\operatorname{ker}(\sigma \rtimes V))=\operatorname{ker}\left(\operatorname{Ind}_{H}^{G}(\sigma \rtimes V)\right)
$$

for any nondegenerate representation $\sigma \rtimes V$ of $A \rtimes_{\alpha} H$, and

$$
\operatorname{Res}(\operatorname{ker}(\pi \rtimes U))=\operatorname{ker} \pi
$$

for any nondegenerate representation $\pi \rtimes U$ of $A \rtimes_{\alpha} G$, where Ind and Res denote induction and restriction of representations. For a primitive ideal $P$ we denote by

$$
G_{P}:=\left\{s \in G: \alpha_{s}(P)=P\right\}
$$

the stability group at $P$ for the continuous action of $G$ on $\operatorname{Prim}(A)$.

Definition 1.1. We say that the dynamical system $(A, G, \alpha)$ satisfies the EffrosHahn induction property (EHI) if, given $P \in \operatorname{Prim}(A)$ and a primitive ideal $J$ in $\operatorname{Prim}\left(A \rtimes_{\alpha} G_{P}\right)$ with $\operatorname{Res} J=P$, then $\operatorname{Ind}_{G_{P}}^{G} J$ is a primitive ideal in $A \rtimes_{\alpha} G$. We say $(A, G, \alpha)$ satisfies the strong Effros-Hahn induction property (strong-EHI) if, given $P \in \operatorname{Prim}(A)$ and an irreducible representation $\rho \rtimes V$ of $A \rtimes_{\alpha} G_{P}$ with $\operatorname{ker} \rho=P$, then $\operatorname{Ind}_{G_{P}}^{G}(\rho \rtimes V)$ is irreducible.

Received by the editors October 25, 2005 and, in revised form, February 12, 2007.

2000 Mathematics Subject Classification. Primary 46L55, 46L05.

The authors were partly supported by the Ed Shapiro fund at Dartmouth College and the Deutsche Forschungsgemeinschaft (SFB 478).

(C)2008 American Mathematical Society Reverts to public domain 28 years from publication 
It is clear that strong-EHI implies EHI. It is well known that all separable systems $(A, G, \alpha)$ with $A$ commutative satisfy strong-EHI. The proof goes back to Mackey (15. §6]; see also [9, pp. 900-901]). In fact, the separability assumption can be dropped by [24, Proposition 4.2]. Building on [24, Proposition 4.2], Olesen and Raeburn were able to show that if $A$ is a separable continuous-trace $C^{*}$-algebra with $G$ acting freely on the spectrum, then $(A, G, \alpha)$ satisfies strong-EHI [17, Lemma 3.2]1] It follows from Green's proof of [11, Theorem 24] that if $(A, G, \alpha)$ is separable with $G$ amenable and acting freely on $\operatorname{Prim} A$, then $(A, G, \alpha)$ satisfies EHI 2 Green's result generalizes a result of Zeller-Meier for $G$ discrete [25, Theorem 5.15]. We would like to believe that the following conjecture is true.

Conjecture 1.2. All separable dynamical systems $(A, G, \alpha)$ satisfy EHI.

If we add the hypothesis that $G$ is amenable, then EHI is claimed as part of the first author's work [6, Theorem 1.4.14]. However, [6, Theorem 1.4.14] is meant to be a summary of the main results in Gootman and Rosenberg [10, and for the result we are interested in, Gootman and Rosenberg are relying on Sauvageot's result [21, Proposition 2.1]. The problem is that Sauvageot does not work with an arbitrary primitive ideal in $A \rtimes_{\alpha} G_{P}$ with restriction $P$. He requires an additional assumption that certain associated representations are homogeneous:

Definition 1.3. A representation $\pi: A \rightarrow B\left(\mathcal{H}_{\pi}\right)$ is called homogeneous if every nonzero subrepresentation of $\pi$ has the same kernel as $\pi$.

Sauvageot's result is as follows.

Proposition 1.4 ([21, Proposition 2.1]). Let $(A, G, \alpha)$ be a separable dynamical system. Suppose that $\rho$ is a homogeneous representation of $A$ with kernel $P$, and that $\rho \rtimes V$ is a homogeneous representation of $A \rtimes_{\alpha} G_{P}$. Then $\operatorname{Ind}_{G_{P}}^{G}(\rho \rtimes V)$ is a homogeneous representation of $A \rtimes_{\alpha} G$.

Remark 1.5. It is clear that every irreducible representation is homogeneous. On the other hand, a representation $\pi: A \rightarrow B\left(\mathcal{H}_{\pi}\right)$ is homogeneous if and only if given $I \in \mathcal{I}(A)$, then $\overline{\pi(I) \mathcal{H}_{\pi}}$ is either all of $\mathcal{H}_{\pi}$ or $\{0\}$. This is the content of [21, Lemme 1.5] or [7, Theorem 1.4]. It follows that the kernel of a homogeneous representation is always a prime ideal [7, Corollary 1.5]. Since every prime ideal is primitive in a separable $C^{*}$-algebra [19, Theorem A.49], the kernel of any homogeneous representation of a separable $C^{*}$-algebra is a primitive ideal 3

Remark 1.6. As the emphasis in Proposition 1.4 indicates, our problem is resolving Sauvageot's "extra" hypothesis on $\rho$ with Conjecture 1.2. Unfortunately, Sauvageot's proof makes significant use of the homogeneity of $\rho$ as it is necessary to view $\operatorname{Ind}_{G_{P}}^{G}(\rho \rtimes V)$ as a direct integral of homogeneous representations over $G / G_{P}$. Gootman and Rosenberg are careful to note this property in their definition of an "induced primitive ideal" just prior to stating their Theorem 3.1 on page 290 of

\footnotetext{
${ }^{1}$ Olesen and Raeburn assumed that $G$ was abelian, but their proof was extended to the nonabelian case by Deicke in [4].

${ }^{2}$ Green's proof requires only that $(A, G, \alpha)$ be what he calls "EH-regular". The GootmanRosenberg-Sauvageot result [10] implies this for separable systems with $G$ amenable. (Note that Ind $I=\operatorname{Ind} \bigcap_{s} s \cdot I$ by [11, Proposition 11(ii)].) The result for free actions on separable systems follows from Proposition 1.9

${ }^{3}$ Separability may be crucial here. Weaver has constructed a nonseparable example of a prime ideal which is not primitive 23 .
} 
[10. Unfortunately, these assumptions were neglected by the first author when he included EHI as part of [6, Theorem 1.4.14], so we do not know whether that result is valid in the full generality as stated there. Fortunately, this does not affect any other results of [6], since Propositions 1.8 and 1.9 stated below clearly imply EHI in all situations where [6, Theorem 1.4.14] was used in that Memoir.

Using techniques similar to Sauvageot's, in 92 we prove the following variation of Proposition 1.4

Theorem 1.7. Let $(A, G, \alpha)$ be a separable dynamical system. Suppose that $\rho$ is a homogeneous representation of $A$ with kernel $P$, and that $\rho \rtimes V$ is an irreducible representation of $A \rtimes_{\alpha} G_{P}$. Then $\operatorname{Ind}_{G_{P}}^{G}(\rho \rtimes V)$ is an irreducible representation of $A \rtimes_{\alpha} G$.

In order to use the above propositions for proving (strong-)EHI, it would certainly be sufficient to show that for any irreducible representation $\rho \rtimes V$ of $A \rtimes_{\alpha} G_{P}$ with $\operatorname{ker} \rho=P$, the representation $\rho$ is automatically homogeneous. Unfortunately, this turns out to be not true in general (see Example 3.4 below). However, we shall see that it is true if $P \in \operatorname{Prim}(A)$ is locally closed; that is, if the point set $\{P\}$ is open in $\overline{\{P\}}$. There are actually many $C^{*}$-algebras with the property that all points in $\operatorname{Prim}(A)$ are locally closed; for example, all type I $C^{*}$-algebras and all group $C^{*}$-algebras of almost connected groups (i.e., groups with cocompact connected component) have this property. As a consequence of this and Theorem 1.7 we get

Proposition 1.8. Suppose that $(A, G, \alpha)$ is a separable dynamical system such that all points are locally closed in $\operatorname{Prim}(A)$. Then $(A, G, \alpha)$ satisfies strong-EHI. In particular, if $(A, G, \alpha)$ is a separable dynamical system such that either $A$ is type $I$ or $A$ is a subquotient of the group $C^{*}$-algebra of an almost connected locally compact group, then $(A, G, \alpha)$ satisfies strong-EHI.

Another positive result towards Conjecture 1.2 is the following (see $\$ 3$ for the proof):

Proposition 1.9. Suppose that $(A, G, \alpha)$ is a separable dynamical system such that $G_{P}$ is normal in $G$ for all $P \in \operatorname{Prim}(A)$ (which is clearly true if $G$ is abelian). Then $(A, G, \alpha)$ satisfies strong-EHI.

If we only consider the weaker property EHI and if we are willing to assume that all stabilizers are amenable, the normality assumption on the stabilizers used above can be weakened to obtain the following result (see 93 ):

Proposition 1.10. Suppose that $(A, G, \alpha)$ is a separable $C^{*}$-dynamical system such that all stabilizers $G_{P}$ are amenable and such that for all $P, Q \in \operatorname{Prim}(A)$ satisfying

$$
P=\bigcap_{s \in G_{Q}} s \cdot Q
$$

we have $G_{Q} \subseteq N\left(G_{P}\right)$ or $G_{P} \subseteq N\left(G_{Q}\right)$ (where $N(H)=\left\{s \in G: s H s^{-1} \subseteq H\right\}$ denotes the normalizer of a subgroup $H$ of $G)$. Then $(A, G, \alpha)$ satisfies EHI.

A provocative program for producing a counterexample to Conjecture 1.2, or to strong-EHI, would involve finding an example of a group action on a $C^{*}$-algebra which does not satisfy the normality condition on the stabilizers as used in the 
above proposition. However, this seems to be difficult. The importance of settling Conjecture 1.2 is discussed in more detail in 4 .

In the appendix, the first author uses this opportunity to give a corrected proof of 6. Theorem 5.5.13]. This is necessary because the original proof relies on [6, Lemma 5.5.17], which turned out to be false.

\section{IRREDUCIBLE INDUCED REPRESENTATIONS}

In this section we want to give the proof of Theorem 1.7 as stated in the introduction. As mentioned there, we will make extensive use of the ideas developed by Sauvageot in 21. Let us first review the process of inducing representations (cf., e.g., [19]). Let $H$ be a closed subgroup of $G$ and let $(\rho, V)$ be a covariant representation of $\left(A, H,\left.\alpha\right|_{H}\right)$ on $\mathcal{H}$. Let $\mathrm{X}=\overline{C_{c}(G, A)}$ be Green's $C_{0}(G / H, A) \rtimes_{\operatorname{lt} \otimes \alpha}$ $G-A \rtimes_{\alpha} H$-imprimitivity bimodule, where lt $: G \rightarrow$ Aut $\left(C_{0}(G / H)\right)$ denotes left translation. Let $\mathrm{X}-\operatorname{Ind}(\rho \rtimes V)$ be the representation of $E:=C_{0}(G / H, A) \rtimes_{\mathrm{lt} \otimes \alpha} G$ corresponding to $\rho \rtimes V$ via $\mathbf{X}$. Then $\mathrm{X}-\operatorname{Ind}(\rho \rtimes V)$ acts on the Hilbert space $\mathcal{V}$, which is the completion of $C_{c}(G, A) \odot \mathcal{H}$ with respect to the pre-inner product

$$
(f \otimes h \mid g \otimes k):=\left(\rho \rtimes V\left(\langle g, f\rangle_{A \rtimes_{\alpha} H}\right) h \mid k\right),
$$

where

$$
\langle g, f\rangle_{A \rtimes_{\alpha} H}(t)=\gamma(t) \int_{G} \alpha_{s}^{-1}\left(g(s)^{*} f(s t)\right) d \mu_{G}(s) \quad \text { and } \quad \gamma(t):=\left(\frac{\Delta_{G}(t)}{\Delta_{H}(t)}\right)^{\frac{1}{2}} .
$$

If $c \in E_{0}:=C_{c}(G \times G / H, A)$, then $(\mathrm{X}-\operatorname{Ind}(\rho \rtimes V))(c)$ sends the class of $f \otimes h$ to the class of $c \cdot f \otimes h$, where

$$
c \cdot f(s):=\int_{G} c(r, \dot{s}) \alpha_{r}\left(f\left(r^{-1} s\right)\right) d \mu_{G}(r) .
$$

The representation $\operatorname{Ind}_{H}^{G}(\rho \rtimes V)$ of $A \rtimes_{\alpha} G$ induced from $\rho \rtimes V$ is, by definition, the restriction of $\mathrm{X}-\operatorname{Ind}(\rho \rtimes V)$ to the image of $A \rtimes_{\alpha} G$ in $M(E)$. Thus, if $g \in$ $C_{c}(G, A) \subseteq A \rtimes_{\alpha} G$, then $\operatorname{Ind}_{H}^{G}(\rho \rtimes V)(g)$ maps the class of $f \otimes h$ to the class of $g * f \otimes h$.

2.1. Realizing induced representations on $L_{V}^{2}(G, \beta ; \mathcal{H})$. We want to realize both these induced representations on the classical space $L_{V}^{2}(G, \beta ; \mathcal{H})$ (cf. [8, §6.1]). As described in [8, $\S 6.1$, Remark 1], we can realize $L_{V}^{2}(G, \beta ; \mathcal{H})$ as the collection of $\mu_{G}$-almost everywhere equivalence classes of functions in $\mathscr{L}_{V}^{2}(G, \beta ; \mathcal{H})$ consisting of Borel functions $\xi: G \rightarrow \mathcal{H}$ such that for all $s \in G$ and $t \in H$,

$$
\xi(s t)=V_{t}^{-1}(\xi(s))
$$

and such that

$$
\int_{G / H}\|\xi(s)\|^{2} d \beta(\dot{s})<\infty
$$

where $\beta$ is a quasi-invariant measure on $G / H$ corresponding to a positive continuous function $\varphi: G \rightarrow(0, \infty)$ such that

$$
\varphi(s t)=\frac{\Delta_{H}(t)}{\Delta_{G}(t)} \varphi(s) \quad \text { for all } s \in G \text { and } t \in H .
$$


$L_{V}^{2}(G, \beta ; \mathcal{H})$ is a Hilbert space with respect to the inner product

$$
(\xi \mid \eta):=\int_{G / H}(\xi(s) \mid \eta(s)) d \beta(\dot{s}) .
$$

We can define $W: C_{c}(G, A) \odot \mathcal{H} \rightarrow \mathscr{L}_{V}^{2}(G, \beta ; \mathcal{H})$ by

$$
W(f \otimes h)(r):=\int_{H} \varphi(r t)^{-\frac{1}{2}} \rho\left(\alpha_{r}^{-1}(f(r t))\right) V_{t} h d \mu_{H}(t) .
$$

Then $W$ extends to a unitary transformation from $\mathcal{V}$ onto $L_{V}^{2}(G, \beta ; \mathcal{H})$.

Any representation of $C_{0}(G / H, A) \rtimes_{1 \mathrm{t} \otimes \alpha} G$ is determined by a covariant representation $(\pi, U)$ on $(A, G, \alpha)$ together with a representation $M$ of $C_{0}(G / H)$ which commutes with $\pi$. It is not hard to check that $W$ intertwines $\mathrm{X}$ - $\operatorname{Ind}(\rho \rtimes V)$ with the representation determined by $\pi, U$ and $M$ given by

$$
\begin{aligned}
M(\phi) \xi(s) & :=\phi(\dot{s}) \xi(s), \\
\pi(a) \xi(s) & :=\rho\left(\alpha_{s}^{-1}(a)\right) \xi(s), \\
U(r) \xi(s) & :=\left(\frac{\varphi\left(r^{-1} s\right)}{\varphi(s)}\right)^{\frac{1}{2}} \xi\left(r^{-1} s\right) .
\end{aligned}
$$

The representation intertwined with $\operatorname{Ind}_{H}^{G}(\rho \rtimes V)$ is given by $\pi \rtimes U$.

2.2. Realizing $\pi$ as a direct integral over $G / H$. We want to see that $\pi$ can be viewed as a very elementary direct integral. Actually, we don't need to invoke direct integrals in their full glory (as described, for example, in [5]). It will be enough to rely on the treatment in [1, §4.2], which considers the special case where the underlying Hilbert space is of the form $L^{2}(X, \mu ; \mathcal{H})$. To apply these results, we need a few observations. Let $c: G / H \rightarrow G$ be a Borel cross section for the quotient map, and let $b: G \rightarrow H$ be the Borel map defined by $b(s)=c(\dot{s})^{-1} s$ for all $s \in G$. Note that $b(s t)=b(s) t$ for all $s \in G$ and $t \in H$. If $f \in \mathscr{L}^{2}(G / H, \beta ; \mathcal{H})$, then

$$
H_{f}(s):=V_{b(s)}^{-1}(f(\dot{s}))
$$

belongs to $\mathscr{L}_{V}^{2}(G, \beta ; \mathcal{H})$ and $\left\|H_{f}\right\|_{2}=\|f\|_{L^{2}(G / H ; \mathcal{H})}$. Conversely, if $h \in$ $\mathscr{L}_{V}^{2}(G, \beta ; \mathcal{H})$, then

$$
F_{h}(\dot{s}):=h(c(\dot{s}))
$$

is in $\mathscr{L}^{2}(G / H, \beta ; \mathcal{H})$. The maps $f \mapsto H_{f}$ and $h \mapsto F_{h}$ are inverses, and we obtain an isomorphism between $L_{V}^{2}(G, \beta ; \mathcal{H})$ and $L^{2}(G / H, \beta ; \mathcal{H})$ 년 Thus we can view $\mathscr{L}_{V}^{2}(G, \beta ; \mathcal{H})$ as the $L^{2}$-sections of the trivial Borel Hilbert bundle $G / H \times \mathcal{H}$. By definition, the diagonal operators $\Delta(G / H \times \mathcal{H}, \beta)$ are naturally identified with $L^{\infty}(G / H)$; if $\psi$ is a bounded Borel function on $G / H$, then the corresponding diagonal operator on $L_{V}^{2}(G, \beta ; \mathcal{H})$ is given by extending $M$ in the obvious way:

$$
M(\psi) \xi(s):=\psi(\dot{s}) \xi(s) .
$$

As shown in [1, Theorem 4.2.1], the decomposable operators are exactly the commutant $M\left(L^{\infty}(G / H)\right)^{\prime}$, and they are identified with equivalence classes of bounded weak-operator Borel functions $F: G / H \rightarrow B(\mathcal{H})$ via

$T_{F}(h)(s):=\widetilde{F}(s)(h(s))$, where $\quad \widetilde{F}(s):=V_{b(s)}^{-1} F(\dot{s}) V_{b(s)} \quad$ and $\quad h \in \mathscr{L}_{V}^{2}(G, \beta ; \mathcal{H})$.

\footnotetext{
${ }^{4}$ This isomorphism also intertwines our version of the induced representations with one in the spirit of the ground breaking work in 22 .
} 
Note that $\tilde{F}$ satisfies

$$
\widetilde{F}(s t)=V_{t}^{-1} \widetilde{F}(s) V_{t} \quad \text { for all } s \in G \text { and } t \in H .
$$

If $\widetilde{F}: G \rightarrow B(\mathcal{H})$ is any weak-operator Borel function satisfying (2.1), then $F(\dot{s}):=$ $\widetilde{F}(c(\dot{s}))$ is a weak-operator Borel function on $G / H$ such that

$$
T_{F} h(s)=\widetilde{F}(s)(h(s)) .
$$

Thus we can identify the decomposable operators on $L_{V}^{2}(G, \beta ; \mathcal{H})$ with the bounded weak-operator Borel functions $F: G \rightarrow B(\mathcal{H})$ that transform as in (2.1). Since

$$
\rho\left(\alpha_{s t}^{-1}(a)\right)=V_{t}^{-1} \rho\left(\alpha_{s}^{-1}(a)\right) V_{t},
$$

$s \mapsto \rho\left(\alpha_{s}^{-1}(a)\right)$ is a bounded weak-operator Borel function transforming as in (2.1). Therefore we can express $\pi$ as a direct integral

$$
\pi=\int_{G / H}^{\oplus} \rho_{\dot{s}} d \beta^{P}(\dot{s})
$$

where

$$
\rho_{\dot{s}}(a) h(\dot{s})=\rho\left(\alpha_{c(\dot{s})}^{-1}(a)\right)(h(\dot{s}))
$$

2.3. The role played by the homogeneity of $\rho$. From here on, we shall ignore the unitary $W$ and identify $\operatorname{Ind}_{H}^{G}(\rho \rtimes V)$ with $(\pi, U)$ and $\mathrm{X}-\operatorname{Ind}(\rho \rtimes V)$ with $((M \otimes$ $\pi), U)$. We will also specialize to the situation where $H=G_{P}$ and $(\rho, V)$ is an irreducible representation with $\rho$ a homogeneous representation with kernel $P$. This assumption on $\rho$ is used to show that all operators in the commutant of $\pi$ are decomposable. This is the essential step in both our proof of Theorem 1.7 and Sauvageot's proof of Proposition 1.4 .

Proof of Theorem 1.7. Decompose $\pi$ as in (2.2). Since $\rho$ is homogeneous with kernel $P$, each $\rho_{\dot{s}}$ is homogeneous with kernel $s \cdot P$. Thus if $\dot{s} \neq \dot{r}$, then $\operatorname{ker} \rho_{\dot{s}} \neq \operatorname{ker} \rho_{\dot{r}}$. Thus (2.3) is an example of the sort of decomposition studied by Effros in [7, and what is now called an ideal center decomposition. One of the major theorems of Effros's theory is that for such a decomposition, the diagonal operators

$$
M\left(L^{\infty}\left(G / G_{P}\right)\right)=\Delta\left(G / G_{P} \times \mathcal{H}, \beta\right)
$$

coincide with a certain von Neumann subalgebra, $\mathcal{I C}(\pi)$, of the center $\pi(A)^{\prime \prime} \cap \pi(A)^{\prime}$ ([7. Theorem 1.10]). Since $M\left(L^{\infty}\left(G / G_{P}\right)\right)=\Delta\left(G / G_{P} \times \mathcal{H}, \beta\right)=\mathcal{I C}(\pi) \subset \pi(A)^{\prime \prime} \cap$ $\pi(A)^{\prime}$, we have

$$
\pi(A)^{\prime} \subset M\left(L^{\infty}\left(G / G_{P}\right)\right)^{\prime} \subset M\left(C_{0}\left(G / G_{P}\right)\right)^{\prime} .
$$

Thus if $T$ is in the commutant of $\operatorname{Ind}_{G_{P}}^{G}(\rho \rtimes V)=\pi \rtimes U$, then $T \in U(G)^{\prime}$ and $T \in \pi(A)^{\prime}$. It follows then from (2.4) that $T \in M\left(C_{0}\left(G / G_{P}\right)\right)^{\prime}$ too. But then $T$ is in the commutant of the irreducible representation $\mathrm{X}-\operatorname{Ind}(\rho \rtimes V)=(M \otimes \pi) \rtimes U$. Hence $T$ is a scalar operator and the commutant of $\operatorname{Ind}_{G_{P}}^{G}(\rho \rtimes V)$ is trivial. Thus $\operatorname{Ind}_{G_{P}}^{G}(\rho \rtimes V)$ is irreducible. 


\section{3. (STRONG-)EHI AND EXAMPLES}

In this section we want to deduce all remaining results as stated in the introduction and present some examples. We start with recording the following elementary consequence of the definition of the Jacobson Topology on $\operatorname{Prim}(A)$.

Lemma 3.1. Suppose that $A$ is a $C^{*}$-algebra and that $P \in \operatorname{Prim}(A)$. Then $\{P\}$ is open in $\overline{\{P\}}$ if and only if $J:=\bigcap\left\{P^{\prime} \in \operatorname{Prim}(A): P^{\prime} \supset P, P^{\prime} \neq P\right\}$ properly contains $P$. (By convention, the intersection over the empty set is the whole algebra A.)

Proof. If $\{P\}$ is open in its closure, then there is an ideal $I \in \mathcal{I}(A)$ such that

$$
\mathcal{O}_{I}:=\left\{P^{\prime} \in \operatorname{Prim}(A): P^{\prime} \not \supset I\right\}
$$

contains $P$ and is disjoint from $\overline{\{P\}} \backslash\{P\}$. But then it follows that $J \supset I$, and $P \not \supset I$ forces $J \neq P$ as required. Conversely, if $J$ properly contains $P$, then $\mathcal{O}_{J}=\left\{P^{\prime} \in \operatorname{Prim}(A): P^{\prime} \not \supset J\right\}$ is an open set containing $P$ and disjoint from $\overline{\{P\}} \backslash\{P\}$.

We use this result to show

Lemma 3.2. Let $(A, G, \alpha)$ be a $C^{*}$-dynamical system and assume that $P \in \operatorname{Prim}(A)$ is locally closed. Suppose that $\rho \rtimes V$ is an irreducible representation of $A \rtimes_{\alpha} G_{P}$ with $\operatorname{ker} \rho=P$. Then $\rho$ is homogeneous.

Proof. In view of Remark 1.5, it will suffice to see that if $I$ is an ideal in $A$ such that $I \not \subset P$, then

$$
\overline{\rho(I) \mathcal{H}_{\rho}}=\mathcal{H}_{\rho} .
$$

Since $\rho(I)=\rho(I+P)$, we can assume that $I$ strictly contains $P$. If $s \in G_{P}$, then $\alpha_{s}(I)$ strictly contains $P$, and since every ideal in $A$ is the intersection of the primitive ideals which contain it, our assumption on $\{P\}$ and Lemma 3.1 imply that

$$
K:=\bigcap_{s \in G_{P}} \alpha_{s}(I) \supset \bigcap_{\substack{P^{\prime} \in \operatorname{Prim}(A) \\ P^{\prime} \supset P \\ P^{\prime} \neq P}} P^{\prime}:=J
$$

properly contains $P$. But $K$ is a $G_{P}$-invariant ideal so that $\overline{\rho(K) \mathcal{H}_{\rho}}$ is nonzero and invariant for both $\rho$ and $V$ : if $a \in K$ and $s \in G_{P}$, then

$$
V_{s} \rho(a) h=\rho\left(\alpha_{s}(a)\right) V_{s} h \in \rho(K) \mathcal{H}_{\rho} .
$$

Since $\rho \rtimes V$ is irreducible by assumption, $\overline{\rho(K) \mathcal{H}_{\rho}}$ must be all of $\mathcal{H}_{\rho}$. Since $\rho(I) \mathcal{H}_{\rho} \supset$ $\rho(K) \mathcal{H}_{\rho}$, this suffices.

Proof of Proposition 1.8. The result will follow directly from Theorem 1.7 together with Lemma 3.2 as soon as we see that if $A$ is type I or if $A$ is a subquotient of $C^{*}(H)$ for some almost connected locally compact group $H$, then points in $\operatorname{Prim}(A)$ are locally closed. If $A$ is type I, then we can identify $\overline{\{P\}}$ with $\operatorname{Prim}(A / P)$, so that we may as well assume that $A$ is primitive (i.e., $\{0\}$ is a primitive ideal), and prove that $\{\{0\}\}$ is open in $\operatorname{Prim}(A)$. Since $A$ is type I, it follows that $A$ must contain an essential ideal $I$ isomorphic to the compact operators on some Hilbert space. In particular, every nonzero ideal in $A$ contains $I$. Now the result follows from Lemma 3.1 
The statement for subquotients of $C^{*}(H)$ for almost connected groups $H$ will follow if we know that points in $\operatorname{Prim}\left(C^{*}(H)\right)$ are locally closed. By [14, Theorem 2.1] we may assume without loss of generality that $H$ is a Lie group with finitely many components. By [16, Theorem 1], we know that the points in $\operatorname{Prim}\left(C^{*}\left(H_{0}\right)\right)$ are locally closed, where $H_{0}$ denotes the connected component of $H$. Moreover, it is shown in [16. Theorem 3] that this property is inherited by $\operatorname{Prim}\left(C^{*}(H)\right)$ if $H / H_{0}$ is solvable. But the solvability condition on $H / H_{0}$ has been removed successfully by Poguntke in [18, (see [18, Satz] together with the discussion following it; notice that a slight modification of the proof of [18, Satz 2] will give this result as a consequence of [18, Satz 1]).

Example 3.3. There are separable $C^{*}$-algebras with primitive ideals $P$ such that $\{P\}$ is not open in $\overline{\{P\}}$. This can even happen for the group $C^{*}$-algebra of countable solvable groups (see the discussion at the end of [16, §3]). For another sort of example, let $A$ be the $C^{*}$-algebra $C^{*}(E)$ associated to the graph $E$ with vertices $E^{0}=\mathbf{Z}^{2}$, and with edges connecting $(i, j)$ to both $(i+1, j)$ and $(i, j+1)$ :

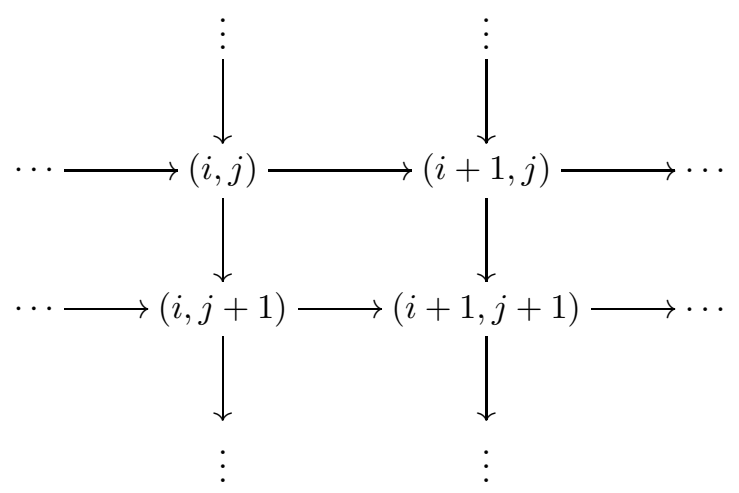

We refer to 3 for the details of the construction of $C^{*}(E)$. (It was Astrid an Huef who pointed out to us that this algebra had an "interesting" primitive ideal structure.)

Since $E$ has no loops, it satisfies condition (K) of [12, and [3, Proposition 6.1] implies that the primitive ideals of $C^{*}(E)$ are in one-to-one correspondence with the set of maximal tails in $E$. Recall that if $\gamma \subset E^{0}$ is a maximal tail, then the corresponding primitive ideal is $I_{H}$, which is associated to the saturated hereditary subset $H:=E^{0} \backslash \gamma$. If $H$ is any saturated hereditary subset in $E^{0}$ (not necessarily corresponding to a maximal tail), then $I_{H}$ is the ideal generated by the projections $p_{v}$ with $v \in H$. Furthermore, $p_{v} \in I_{H}$ if and only if $v \in H$. (For references and further discussion, see [3, §4].) It is not hard to see that the maximal tails in $E$ are $E^{0}$ itself together with

$$
\gamma_{n}:=\{(i, j): j \geq n\} \quad \text { and } \quad \zeta_{m}:=\{(i, j): i \geq m\} .
$$

In particular, $\gamma_{n}$ corresponds to the primitive ideal $I_{H_{n}}$ associated to

$$
H_{n}:=\{(i, j): j<n\},
$$

and $E^{0}$ corresponds to the zero ideal. (So $C^{*}(E)$ is primitive.) Now

$$
I:=\bigcap_{n=1}^{\infty} I_{H_{-n}}
$$


is an ideal which contains none of the projections $p_{(i, j)}$. Thus, $I=\{0\}$ and Lemma 3.1 implies that $\{\{0\}\}$ is not open in $\operatorname{Prim}(A)$.

Indeed, the above example may be extended to give an example of a $C^{*}$-dynamical system $(A, G, \alpha)$, a primitive ideal $P \in \operatorname{Prim}(A)$, and an irreducible representation $\rho \rtimes V$ of $A \rtimes_{\alpha} G_{P}$ with $\operatorname{ker} \rho=P$, such that $\rho$ is not homogeneous:

Example 3.4. Let $A=C^{*}(E)$ where $E$ is the directed graph of Example 3.3. The universal property of graph algebras guarantees that there is an automorphism $\phi$ of $C^{*}(E)$ which takes the projection $p_{(i, j)}$ to $p_{(i+1, j)}$. Thus in the notation of Example 3.3, we have

$$
\phi\left(I_{H_{n}}\right)=I_{H_{n+1}} .
$$

Let

$$
Q:=I_{H_{0}} \quad \text { and } \quad P:=\{0\}=\bigcap_{n \in \mathbf{Z}} n \cdot Q .
$$

Then $P$ and $Q$ are primitive ideals in $C^{*}(E)$ and we have

$$
G_{Q}=\{e\} \quad \text { and } \quad G_{P}=\mathbf{Z} .
$$

Let $\rho \in C^{*}(E)^{\wedge}$ be such that $\operatorname{ker} \rho=Q$. Then Theorem 1.7 implies that

$$
\operatorname{Ind}_{\{e\}}^{G} \rho
$$

is an irreducible representation of $C^{*}(E) \rtimes_{\phi} \mathbf{Z} 5$ Since $G_{P}=\mathbf{Z}$, it follows that $\pi \rtimes U:=\operatorname{Ind}_{\{e\}}^{G} \rho$ is an irreducible representation of $C^{*}(E) \rtimes_{\phi} G_{P}$ such that $\pi \cong \bigoplus_{n \in \mathbf{Z}}\left(\phi^{n} \circ \rho\right)$ and

$$
\operatorname{ker} \pi=\bigcap_{n \in \mathbf{Z}} \operatorname{ker}\left(\phi^{n} \circ \rho\right)=\bigcap_{n \in \mathbf{Z}} n \cdot Q=\{0\}=P .
$$

Since $\rho$ is a subrepresentation of $\pi$ with $\operatorname{ker} \rho=Q \neq P=\operatorname{ker} \pi$ it follows that $\pi$ is not homogeneous.

One might expect that the failure of homogeneity of $\rho$ for an irreducible representation $\rho \rtimes V$ of $A \rtimes_{\alpha} G_{P}$ with ker $\rho=P$ might also lead to a counterexample for (strong-)EHI. This idea certainly doesn't work in the above example, since there we have $G_{P}=G$. Indeed, Proposition 1.9, which we are now going to prove, shows that in order to get a counterexample to strong-EHI, one has to consider actions of nonabelian groups:

Proof of Proposition 1.9. Suppose that $P \in \operatorname{Prim}(A)$ and that $\rho \rtimes V$ is an irreducible representation of $A \rtimes_{\alpha} G_{P}$ with $\operatorname{ker} \rho=P$. Since $G_{P}$ is normal in $G$, it follows from [11, Proposition 1] (see also [6, Example 1.1.1]) that there is a natural isomorphism

$$
A \rtimes_{\alpha} G \cong\left(A \rtimes_{\alpha} G_{P}\right) \rtimes_{\gamma}^{\tau} G
$$

for a twisted system $\left(A \rtimes_{\alpha} G_{P}, G, \gamma, \tau\right)$, where (following the notation in [6])

for $f \in C_{c}\left(G_{P}, A\right)$,

$$
\gamma_{s}(f)(t):=\delta(s) \alpha_{s}\left(f\left(s^{-1} t s\right)\right)
$$

$$
\int_{G_{P}} g\left(s t s^{-1}\right) d \mu_{G_{P}}(t)=\delta(s) \int_{G_{P}} g(t) d \mu_{G_{P}}(t)
$$

\footnotetext{
${ }^{5}$ Note that by [13, Theorem 1.1], $C^{*}(E) \rtimes_{\phi} \mathbf{Z} \cong \mathcal{K}$.
} 
for $g \in C_{c}\left(G_{P}\right)$, and

$$
\tau(t)(f)(s)=\alpha_{t}\left(f\left(t^{-1} s\right)\right)
$$

for $f \in C_{c}\left(G_{P}, A\right)$ and $t \in G_{P}$. Similarly, we have an isomorphism

$$
A \rtimes_{\alpha} G_{P} \cong\left(A \rtimes_{\alpha} G_{P}\right) \rtimes_{\gamma}^{\tau} G_{P},
$$

and this isomorphism intertwines $\rho \rtimes V$ with $(\rho \rtimes V) \rtimes V$. In particular, $(\rho \rtimes V) \rtimes V$ is irreducible. Furthermore, [6, Proposition 1.4.3] implies that $\operatorname{Ind}_{G_{P}}^{G}((\rho \rtimes V) \rtimes V)$ is intertwined with $\operatorname{Ind}_{G_{P}}^{G}(\rho \rtimes V)$ by the isomorphism of (3.1). Therefore it will suffice to show that

$$
\operatorname{Ind}_{G_{P}}^{G}((\rho \rtimes V) \rtimes V)
$$

is irreducible. To prove this, we want to apply Theorem 1.7 to the dynamical system $\left(A \rtimes_{\alpha} G_{P}, G, \gamma\right)$ and the irreducible representation $(\rho \rtimes V) \rtimes V$. Since $\rho \rtimes V$ is irreducible, and therefore homogeneous, we only need to check that $G_{P}$ is equal to $G_{J}$, where $J$ is equal to $\operatorname{ker}(\rho \times V)$ and $G_{J}$ is the stability group of $J \in \operatorname{Prim}\left(A \rtimes_{\alpha} G_{P}\right)$ with respect to the action induced by $\gamma$. However, as $\gamma$ admits a twist $\tau$ with $N_{\tau}=G_{P}$, we have $G_{P} \subset G_{J}$. On the other hand, it is not hard to check that

$$
(\rho \rtimes V) \circ \gamma_{s}^{-1}=s \cdot \rho \rtimes{ }^{s} V,
$$

where, as usual, $s \cdot \rho:=\rho \circ \alpha_{s}^{-1}$ and ${ }^{s} V(t):=V\left(s t s^{-1}\right)$. In particular,

$$
\operatorname{Res}\left(\gamma_{s}(J)\right)=\alpha_{s}(P) .
$$

Thus $\gamma_{s}(J) \neq J$ if $s \notin G_{P}$. This completes the proof.

3.1. A method in search of a counterexample. Here we see that using the positive solution of the Effros-Hahn conjecture for amenable groups, the "GootmanRosenberg-Sauvageot-Theorem" ([10, Theorem 3.1]), we can obtain a quasi-result. We are not sure whether this result should be viewed as evidence to support Conjecture 1.2 or whether it indicates we should search for a counterexample.

Anyway, suppose that $(A, G, \alpha)$ is a separable dynamical system and that for each $P \in \operatorname{Prim}(A), G_{P}$ is amenable. Fix $J \in \operatorname{Prim}\left(A \rtimes_{\alpha} G_{P}\right)$ with Res $J=P$. Since $G_{P}$ is amenable, we can apply the GRS-Theorem to $\left(A, G_{P}, \alpha\right)$, and conclude that there is a $Q \in \operatorname{Prim}(A)$ and a $K \in \operatorname{Prim}\left(A \rtimes_{\alpha}\left(G_{P}\right)_{Q}\right)$ such that $K=\operatorname{ker}(\sigma \rtimes W)$ with both $\sigma \rtimes W$ and $\sigma$ homogeneous, and with $\operatorname{ker} \sigma=Q$, and such that $\operatorname{Ind}_{\left(G_{P}\right)_{Q}}^{G_{P}} K=J$. Notice that we must have

$$
P=\bigcap_{s \in G_{P}} s \cdot Q
$$

Furthermore,

$$
\left(G_{P}\right)_{Q}=G_{Q} \cap G_{P}
$$

Now if $G_{Q} \subset G_{P}$, then $\left(G_{P}\right)_{Q}=G_{Q}$ and we have

$$
\begin{aligned}
\operatorname{Ind}_{G_{P}}^{G} J & =\operatorname{Ind}_{G_{P}}^{G}\left(\operatorname{ker}\left(\operatorname{Ind}_{G_{Q}}^{G_{P}} \sigma \rtimes W\right)\right) \\
& =\operatorname{ker}\left(\operatorname{Ind}_{G_{Q}}^{G} \sigma \rtimes W\right) .
\end{aligned}
$$


It follows that (3.4) is primitive by Sauvageot's Proposition 1.4. Thus we obtain the following awkwardly stated result:

Lemma 3.5. Suppose that $(A, G, \alpha)$ is a separable dynamical system such that $G_{P}$ is amenable for all $P \in \operatorname{Prim}(A)$. Suppose that for all $P, Q \in \operatorname{Prim}(A)$ such that (3.3) holds, we have $G_{Q} \subset G_{P}$. Then $(A, G, \alpha)$ satisfies EHI.

Remark 3.6. If (3.3) holds, then we have $G_{Q} \subset G_{P}$ provided either

$$
G_{Q} \subset N\left(G_{P}\right) \text { or } G_{P} \subset N\left(G_{Q}\right) \text {, }
$$

where

$$
N(H)=\left\{s \in G: s H s^{-1}=H\right\} .
$$

So the above lemma clearly implies Proposition 1.10

\section{The Problem With the CURRent State of the ART}

The positive solution of the Effros-Hahn conjecture due to Gootman and Rosenberg [10, building on the work of Sauvageot 20, 21, is a critical ingredient in describing the fine ideal structure of a separable crossed product by an amenable group. Nevertheless, the current state of the art leaves something to be desired. In order to describe the fine ideal structure of a $C^{*}$-algebra $A$, there are two basic steps. First it is necessary to $\operatorname{describe} \hat{A}$ or $\operatorname{Prim}(A)$ as a set. Secondly, we want to describe the hull-kernel topology. Both steps can be formidable. For many $C^{*}$ algebras, especially non-type-I $C^{*}$-algebras, describing $\hat{A}$ can be impossible, and $\operatorname{Prim}(A)$ nearly so. The general procedure for describing either space is to first describe a relatively nice set $X$ consisting of representations (often called concrete representations) and then exhibit either $\hat{A}$ or $\operatorname{Prim}(A)$ as a quotient of $X$. One approach to the second step is to equip $X$ with a topology and show that the hull-kernel topology is the quotient topology.

In the case of crossed products $A \rtimes_{\alpha} G$, a useful example is the case where $G$ is abelian and $A=C_{0}(\hat{A})$. Then a variation on Theorem 1.7 produces a continuous map on $\hat{A} \times \widehat{G}$ into $\operatorname{Prim}\left(A \rtimes_{\alpha} G\right)$. The hard part is to see that this map is surjective, but this is implied by the Gootman-Rosenberg-Sauvageot result. It is shown in 24] that this map factors through the quotient

$$
(\hat{A} \times \widehat{G}) / \sim,
$$

where $(x, \sigma) \sim(y, \rho)$ if $\overline{G \cdot x}=\overline{G \cdot y}$ and $\sigma \bar{\rho} \in G_{x}^{\perp}=G_{y}^{\perp}$, and that we can identify $\operatorname{Prim}\left(C_{0}(\hat{A}) \rtimes G\right)$ with (4.1) as a topological space [24, Theorem 5.3].

If $A$ is CCR, then we can at least identify a candidate for the set $X$ of concrete representations as follows. Given $P \in \operatorname{Prim}(A), A / P$ is simple and we can take any irreducible representation of $A / P \rtimes_{\alpha} G_{P}$ and lift it to an irreducible representation $L_{P}$ of $A \rtimes_{\alpha} G_{P}$ with the property that $\operatorname{Res}\left(\operatorname{ker} L_{P}\right)=P$. Thus by Proposition 1.8, $\operatorname{Ind}_{G_{P}}^{G}\left(\operatorname{ker} L_{P}\right)$ is primitive. If $G$ is amenable, then this gives us a map of

$$
\bigcup_{P \in \operatorname{Prim}(A)} \operatorname{Prim}\left(A / P \rtimes_{\alpha} G_{P}\right)
$$

into $\operatorname{Prim}\left(A \rtimes_{\alpha} G\right)$, which is surjective by the GRS Theorem.

If $A$ is merely GCR, Proposition 1.8 still applies, but the picture gets just a bit more complicated. For any given $P \in \operatorname{Prim}(A), A / P$ contains a simple essential ideal $K(P)$ which is an elementary $C^{*}$-algebra. Any irreducible representation $L$ 
of $K(P) \rtimes_{\alpha} G_{P}$ has a canonical extension $\bar{L}=\rho \rtimes V$ to $A / P \rtimes_{\alpha} G_{P}$ with $\rho$ faithful. Thus we can replace (4.2) by

$$
\bigcup_{P \in \operatorname{Prim}(A)} \operatorname{Prim}\left(K(P) \rtimes_{\alpha} G_{P}\right) .
$$

So even in the GCR case, we have an " $X$ " from which to start. One might even be able to endow $X$ with some sort of topology via a Fell subgroup algebra construction or some groupoid variation. A much more sophisticated and cleaner description is given in [6, Theorem 3.1.7 and Remark 3.1.8].

In the general case, the additional homogeneity hypothesis puts some serious holes in our approach. Even if $G$ is amenable, given $P \in \operatorname{Prim}(A)$, we do not see any reason why there has to be a $J \in \operatorname{Prim}\left(A \rtimes_{\alpha} G_{P}\right)$ with $\operatorname{Res} J=P$. All the existing theory guarantees is that given $K \in \operatorname{Prim}\left(A \rtimes_{\alpha} G\right)$, then there is a $P \in \operatorname{Prim}(A)$ and a $J \in \operatorname{Prim}\left(A \rtimes_{\alpha} G_{P}\right)$ such that Res $J=P$, and of course, $\operatorname{Ind}_{G_{P}}^{G} J=K$. Knowing this is a remarkably powerful tool and certainly gives us plenty of information about $\operatorname{Prim}\left(A \rtimes_{\alpha} G\right)$. It is, for example, very useful in determining the simplicity of $A \rtimes_{\alpha} G$. But it definitely does not provide us with a nice set $X$ in the spirit of (4.1) or (4.2).

\section{Appendix: A corrected proof of [6, Theorem 5.5.13]}

In this appendix, the first author wants to fill a gap in the proof of Theorem 5.5.13 of the Memoir [6], one of the main results of that Memoir. The gap is due to a false lemma ([6, Lemma 5.5.17]), which was used in the original proof. Below, as in [6, $\mathfrak{K}(G)$ denotes the set of closed subgroups of $G$ equipped with Fell's topology. $\mathfrak{K}(G)$ is a compact Hausdorff space, and a base of the topology is given by the sets

$$
U(\mathcal{F}, C)=\{H \in \mathfrak{K}(G) ; H \cap V \neq \emptyset \text { for all } V \in \mathcal{F} \text { and } H \cap C=\emptyset\},
$$

where $\mathcal{F}$ runs through all finite families of open subsets of $G$ and $C$ runs through the compact subsets of $G$.

In [6. Lemma 5.5.17] we stated that if $N$ is an open normal subgroup of $G$ and if $q: G \rightarrow G / N$ denotes the quotient map, then the map $H \rightarrow q(H) ; \mathfrak{K}(G) \rightarrow \mathfrak{K}(G / N)$ is continuous. Unfortunately, this is not true in general. To see a counterexample let $G=\mathbf{Z}$ and $N=2 \mathbf{Z}$. Then, if $H_{n}=(2 n+1) \mathbf{Z}$ we have $H_{n} \rightarrow\{0\}$ in $\mathfrak{K}(\mathbf{Z})$ but $q\left(H_{n}\right)=\mathbf{Z} / 2 \mathbf{Z} \rightarrow \mathbf{Z} / 2 \mathbf{Z}$ in $\mathfrak{K}(\mathbf{Z} / 2 \mathbf{Z})$. A similar example shows that (contrary to the statement of the lemma), if $K$ is a compact subgroup of an abelian group $G$, then the intersection map $H \rightarrow H \cap K ; \mathfrak{K}(G) \rightarrow \mathfrak{K}(K)$ is not continuous in general.

In what follows we want to correct the proof of [6, Theorem 5.5.13]. Note that this was the only place in the Memoir where the lemma was used!

Recall that if $G$ is an abelian locally compact group and $[\omega] \in H^{2}(G, \mathbb{T})$, then $[\omega]$ determines a homomorphism $h_{\omega}: G \rightarrow \widehat{G}$ by $h_{\omega}(s)(t)=\omega(s, t) \omega(t, s)^{-1}$. The group kernel $\Sigma_{\omega}$ of $h_{\omega}$ is called the symmetrizer of $[\omega]$. By a result of Baggett and Kleppner [2, Corollary to Theorem 3.2 and following discussion], it is known that the twisted group algebra $C^{*}(G, \omega)$ is type I if and only if $h_{\omega}$ has closed range and is open as a map onto its image, in which case it factors through an isomorphism between $G / \Sigma_{\omega}$ and $\Sigma_{\omega}^{\perp}$. We then say that $[\omega]$ is type $I$ (see the Memoir for more details). As indicated in the original proof of [6, Theorem 5.5.13], by passing to a Morita equivalent untwisted action and by the definition of the Mackey obstruction 
map $h^{\alpha, \tau}$ as given in [6, Definition 5.5.1], the proof of [6. Theorem 5.5.13] will follow from

Theorem 5.1. Suppose that $(A, G, \alpha)$ is a separable $C^{*}$-dynamical system such that $A$ has continuous trace and $G$ is a compactly generated abelian Lie group. Suppose further that $G$ acts trivially on $\hat{A}$ and for each $x \in \Omega:=\hat{A}$ let $\left[\omega_{x}\right] \in H^{2}(G, \mathbb{T})$ denote the Mackey obstruction at $x$ for extending the corresponding representation $\rho_{x}$ to a covariant representation of $(A, G, \alpha)$. Let

$$
h^{\alpha}: \Omega \times G \rightarrow \Omega \times \widehat{G}, h^{\alpha}(x, s)=\left(x, h_{\omega_{x}}(s)\right) .
$$

Then $h^{\alpha}$ has closed range and is open as a map onto its image if and only if all Mackey obstructions $\left[\omega_{x}\right]$ are type $I$ and the symmetrizer map $\Omega \rightarrow \mathfrak{K}(G), x \mapsto$ $\Sigma_{x}:=\Sigma_{\omega_{x}}$ is continuous.

As a consequence of this result (i.e., 6, Theorem 5.5.13]) and 6, Theorem 5.5.2] it follows that if $(A, G, \alpha)$ is as above, then the crossed product $A \rtimes_{\alpha} G$ has continuous trace if and only if all Mackey obstructions are type I and the symmetrizer map is continuous (but see [6, Corollary 5.5.14] for a more general consequence).

We will need the following basic result, which was also partly stated in 6. Lemma 5.5.17].

Lemma 5.2. Suppose that $G$ is a locally compact group. If $N$ is an open subgroup of $G$ then $\cap_{N}: \mathfrak{K}(G) \rightarrow \mathfrak{K}(N) ; H \mapsto H \cap N$ is continuous. Moreover, if $N$ is any closed normal subgroup of $G$, and if $\mathfrak{K}(G)_{N}$ denotes the set $\{H \in \mathfrak{K}(G): N \subseteq H\}$, then $q_{*}: \mathfrak{K}(G)_{N} \rightarrow \mathfrak{K}(G / N) ; H \mapsto q(H)$ is a homeomorphism.

Proof. Let $H \in \mathfrak{K}(G)$, let $U_{1}, \ldots, U_{l}$ be open subsets of $N$, and let $C \subseteq N$ be compact. Since $N$ is open in $G$, the sets $U_{1}, \ldots, U_{l}$ are also open in $G$. If we denote by $W$ the neighborhood of $H$ in $\mathfrak{K}(G)$ and by $V$ the neighborhood of $H \cap N$ in $\mathfrak{K}(N)$ determined by these sets, then $\cap_{N}(W) \subseteq V$, which proves the continuity of $\cap_{N}$.

To see the second assertion let $U_{1}, \ldots, U_{l}$ be open subsets of $G / N$, and let $C \subseteq$ $G / N$ be compact. Choose a compact subset $K$ of $G$ such that $q(K)=C$. Then

$$
q_{*}\left(U\left(q^{-1}\left(U_{1}\right), \ldots, q^{-1}\left(U_{l}\right), K\right) \cap \mathfrak{K}(G)_{N}\right)=U\left(U_{1}, \ldots, U_{l}, C\right) .
$$

Since all elements of $\mathfrak{K}(G)_{N}$ contain $N$ as a subgroup, every basic open set of $\mathfrak{K}(G)_{N}$ can be written as above. Thus, since $q_{*}$ is bijective, the result follows.

Recall that every closed subgroup $H$ of a direct product $V \times Z$ of a vector group $V$ with a finitely generated free abelian group $Z$ can be written as a direct product $H=W \times S$ of a vector subgroup $W$ of $V$ and a subgroup $S$ of $V \times Z$ which is a finitely generated free abelian group. Thus, for any closed subgroup $H$ of $V \times Z$ there are two characteristic positive integers, namely the dimension, $\operatorname{dim} H:=\operatorname{dim} W$, and the $\operatorname{rank}, \operatorname{rank} H:=\operatorname{rank} S$. The following is [6, Lemma 5.5.18].

Lemma 5.3. Let $V$ be a vector group, and let $H_{n} \rightarrow H$ in $\mathfrak{K}(V)$. If $\operatorname{rank} H \geq$ $\operatorname{rank} H_{n}$ for all $n \in \mathbb{N}$, then there exists a subsequence $\left(H_{n_{k}}\right)_{k \in \mathbb{N}}$ of $\left(H_{n}\right)_{n \in \mathbb{N}}$ and elements $c_{k} \in \mathrm{GL}(V)$ such that $c_{k} \rightarrow \mathrm{id}_{V}$ and $c_{k}(H)=H_{n_{k}}$ for all $k \in \mathbb{N}$.

If $L$ is a compactly generated abelian Lie group, then $L$ has a (noncanonical) splitting $L=W \times T \times S \times F$, where $W$ is a vector group (i.e., isomorphic to some $\left.\mathbb{R}^{n}\right), T$ is a finite-dimensional torus group, $S$ is finitely generated free abelian, and 
$F$ is finite. Although the splitting is noncanonical, the values $\operatorname{dim} V, \operatorname{dim} T, \operatorname{rank} Z$, and the (isomorphism class of the) finite group $F$ form a complete invariant of the isomorphism class of $L$. We need

Lemma 5.4. Let $\Sigma$ be a closed subgroup of the direct product $V \times Z$ of some vector group $V$ with some finitely generated free abelian group $Z$. Let $q: V \times Z \rightarrow Z$ denote the quotient map and let $W \times T \times S \times F$ be a splitting of the compactly generated Lie group $G / \Sigma$ as above. Then $\operatorname{dim} T=\operatorname{rank}(V \cap \Sigma)$ and $\operatorname{rank} S=$ $\operatorname{rank} Z-\operatorname{rank} q(\Sigma)$. In particular, if $G / \Sigma$ is isomorphic to $\Sigma^{\perp} \cong(G / \Sigma)^{\wedge}$, then $\operatorname{rank}(V \cap \Sigma)=\operatorname{rank} Z-\operatorname{rank} q(\Sigma)$.

Proof. Since $V \cdot \Sigma$ is open in $G$ and $q: V \rightarrow V \cdot \Sigma / \Sigma$ factors through an isomorphism between $V /(V \cap \Sigma)$ and $V \cdot \Sigma / \Sigma$, we see that the connected component $W \times T$ of $G / \Sigma$ is isomorphic to $V /(V \cap \Sigma)$. Since the dimension of the maximal torus in $V /(V \cap \Sigma)$ is equal to the rank of $V \cap \Sigma$, it follows that $\operatorname{dim} T=\operatorname{rank}(V \cap \Sigma)$. Further, we have

$$
S \times F \cong(G / \Sigma) /(G / \Sigma)_{0}=(G / \Sigma) /(V \cdot \Sigma / \Sigma) \cong G / V \cdot \Sigma \cong Z / q(\Sigma),
$$

which implies that $\operatorname{rank} S=\operatorname{rank} Z-\operatorname{rank} q(\Sigma)$. The final assertion follows from the fact that if $G / \Sigma$ is isomorphic to its dual group, then $\operatorname{dim} T=\operatorname{rank} S$.

The next lemma closes the main gap in the proof of [6, Theorem 5.5.13].

Lemma 5.5. Let $G=V \times Z$ be a direct product of a vector group $V$ with a finitely generated free abelian group $Z$. Suppose that $\left(\Sigma_{n}\right)_{n \in \mathbb{N}}$ is a sequence in $\mathfrak{K}(G)$ which converges to $\Sigma \in \mathfrak{K}(G)$ such that $G / \Sigma_{n} \cong \Sigma_{n}^{\perp}$ for all $n \in \mathbb{N}$, and such that $G / \Sigma \cong \Sigma^{\perp}$. Then there exists a subsequence of $\left(\Sigma_{n}\right)_{n \in \mathbb{N}}$ (also denoted $\left(\Sigma_{n}\right)_{n \in \mathbb{N}}$ below) which satisfies

(1) there exist elements $c_{n} \in \mathrm{GL}(V)$ such that $c_{n} \rightarrow \mathrm{id}_{V}$ in $\mathrm{GL}(V)$ and such that $V \cap \Sigma_{n}=c_{n}(V \cap \Sigma)$ for all $n \in \mathbb{N}$,

(2) $q\left(\Sigma_{n}\right)=q(\Sigma)$ for all $n \in \mathbb{N}$, where $q: G \rightarrow Z$ denotes the quotient map.

Proof. We first claim that $\operatorname{rank} q\left(\Sigma_{n}\right) \geq \operatorname{rank} q(\Sigma)$ for almost all $n \in \mathbb{N}$. To see this let $s_{1}, \ldots, s_{l} \in \Sigma$ such that $q\left(s_{1}\right), \ldots, q\left(s_{l}\right)$ is a minimal set of generators for $q(\Sigma)$. Since $\Sigma_{n} \rightarrow \Sigma$, it follows from the definition of the topology on $\mathfrak{K}(G)$ that (after passing to a subsequence if necessary) there exist sequences $\left(s_{n}^{i}\right)_{n \in \mathbb{N}}, 1 \leq i \leq l$ such that $s_{n}^{i} \in \Sigma_{n}$ and $s_{n}^{i} \rightarrow s_{i}$ for all $1 \leq i \leq l$. But then $q\left(s_{n}^{i}\right) \rightarrow q\left(s_{i}\right)$ in $Z$ for all $1 \leq i \leq l$, and since $Z$ is discrete we have $q\left(s_{n}^{i}\right)=q\left(s_{i}\right)$ for all $i$ and $n \geq n_{0}$. Thus $q(\Sigma) \subseteq q\left(\Sigma_{n}\right)$ for almost all $n \in \mathbb{N}$, which proves the claim.

Applying the last assertion of Lemma 5.4 we now get

$$
\operatorname{rank}(V \cap \Sigma)=\operatorname{rank} Z-\operatorname{rank} q(\Sigma) \geq \operatorname{rank} Z-\operatorname{rank} q\left(\Sigma_{n}\right)=\operatorname{rank}\left(V \cap \Sigma_{n}\right) .
$$

Thus we may apply Lemma 5.3 to the sequence $V \cap \Sigma_{n}$ (which converges to $V \cap \Sigma$ by Lemma 5.2) in order to see (after passing again to a subsequence) that there exists a sequence $c_{n} \in \mathrm{GL}(V)$ with $c_{n} \rightarrow \operatorname{id}_{V}$ and $c_{n}(V \cap \Sigma)=V \cap \Sigma_{n}$ for all $n$.

The only thing which remains to be shown is the assertion that $q(\Sigma)=q\left(\Sigma_{n}\right)$ for all $n$. It follows from $c_{n}(V \cap \Sigma)=V \cap \Sigma_{n}$ that $\operatorname{rank}\left(V \cap \Sigma_{n}\right)=\operatorname{rank}(V \cap \Sigma)$ for all $n \in \mathbb{N}$, and therefore

$$
\operatorname{rank} q(\Sigma)=\operatorname{rank} Z-\operatorname{rank}(V \cap \Sigma)=\operatorname{rank} Z-\operatorname{rank}\left(V \cap \Sigma_{n}\right)=\operatorname{rank} q\left(\Sigma_{n}\right)
$$

for all $n \in \mathbb{N}$. We embed $Z$ as a discrete subgroup of a vector group $W$, so that we may view all subgroups of $G$ as subgroups of the bigger group $V \times W$. Since 
$\operatorname{rank} \Sigma_{n}=\operatorname{rank}\left(V \cap \Sigma_{n}\right)+\operatorname{rank} q\left(\Sigma_{n}\right)$ for all $n$, and the same equation holds for $\Sigma$, we have $\operatorname{rank} \Sigma_{n}=\operatorname{rank} \Sigma$ for all $n \in \mathbb{N}$. Thus we may apply Lemma 5.3 again in order to obtain elements $d_{n} \in \mathrm{GL}(V \times W)$ such that $d_{n} \rightarrow \operatorname{id}_{V \times W}$ and $\Sigma_{n}=d_{n}(\Sigma)$ for all $n \in \mathbb{N}$. Now let $Y \times S$ be a splitting of $\Sigma$ in its vector group part $Y$ and a finitely generated free abelian part $S$. Let $s_{1}, \ldots, s_{r}$ be a basis of $Y$ and let $s_{r+1}, \ldots, s_{l}$ be a set of generators for $S$. Then each $\Sigma_{n}$ has the splitting $\Sigma_{n}=d_{n}(Y) \times d_{n}(S)$ with generating elements $\left\{d_{n}\left(s_{1}\right), \ldots, d_{n}\left(s_{l}\right)\right\}$. Let $q$ also denote the projection from $V \times W$ to $W$. Then $\left\{q\left(d_{n}\left(s_{1}\right)\right), \ldots, q\left(d_{n}\left(s_{l}\right)\right)\right\}$ is a set of generators of $q\left(\Sigma_{n}\right) \subseteq Z$ for all $n \in \mathbb{N}$. Since $d_{n} \rightarrow \operatorname{id}_{V \times W}$ it follows that $q\left(d_{n}\left(s_{i}\right)\right) \rightarrow q\left(s_{i}\right)$ for each $1 \leq i \leq l$, which implies, since $Z$ is discrete, that they eventually coincide. But this proves that $q\left(\Sigma_{n}\right)=q(\Sigma)$ for all but finitely many $n \in \mathbb{N}$.

Proof of Theorem 5.1. By [6, Lemma 5.5.6] it remains to show that the continuity of the symmetrizer map and the type I property of the Mackey obstructions implies that $h^{\alpha}$ is open as a map onto its image. Each compactly generated Lie group is a quotient of a group $V \times Z$ by some discrete subgroup $D$, where $V$ is a vector group and $Z$ is a finitely generated free abelian group. Thus, by extending the Mackey obstructions to this covering group, we assume without loss of generality that $G=V \times Z$.

Let $\left(x_{n}, s_{n}\right)_{n \in \mathbb{N}}$ be a sequence in $\Omega \times G$ and $(x, s) \in \Omega \times G$ such that $h^{\alpha}\left(x_{n}, s_{n}\right) \rightarrow$ $h^{\alpha}(x, s)$ in $\Omega \times \widehat{G}$. We have to show that, after passing to a subsequence if necessary, there exist elements $t_{n} \in \Sigma_{x_{n}}$ such that $\left(x_{n}, t_{n} s_{n}\right) \rightarrow(x, s)$ in $\Omega \times G$. It is clear that $x_{n} \rightarrow x$ in $\Omega$. Let $q: G \rightarrow G / V \cong Z$ denote the quotient map. Since $\Sigma_{x_{n}} \rightarrow \Sigma_{x}$ in $\mathfrak{K}(G)$ and since $s \mapsto h^{\alpha}\left(x_{n}, s\right)$ factors through an isomorphism between $G / \Sigma_{x_{n}}$ and $\Sigma_{x_{n}}^{\perp}$ (and, similarly, $G / \Sigma_{x} \cong \Sigma_{x}^{\perp}$ ) by the type I assumption on the cocycles, we may apply Lemma 5.5 in order to see that (at least for a subsequence) $q\left(\Sigma_{x_{n}}\right)=q\left(\Sigma_{x}\right)$ for all $n \in \mathbb{N}$, and to obtain a sequence $c_{n} \in \mathrm{GL}(V)$ such that $c_{n} \rightarrow \mathrm{id}_{V}$ and $V \cap \Sigma_{x_{n}}=c_{n}\left(V \cap \Sigma_{x}\right)$ for all $n \in \mathbb{N}$.

For each $n \in \mathbb{N}$ we now define elements $\sigma_{n} \in \operatorname{Aut}(G)$ by $\sigma_{n}=c_{n} \times \operatorname{id}_{Z}$, where $\operatorname{id}_{Z}$ denotes the identity on $Z$. Then $\sigma_{n} \rightarrow \operatorname{id}_{G}$ in $\operatorname{Aut}(G)$ with respect to the compact open topology. Moreover, we have

$$
\sigma_{n}\left(\Sigma_{x} \cap V\right)=\Sigma_{x_{n}} \cap V \quad \text { and } \quad q\left(\sigma_{n}\left(\Sigma_{x}\right)\right)=q\left(\Sigma_{x_{n}}\right)
$$

for all $n \in \mathbb{N}$. By passing to a subsequence, we may assume without loss of generality that $x_{n} \neq x_{m} \neq x$ for all $n, m \in \mathbb{N}$. Let $M=\left\{x_{n} ; n \in \mathbb{N}\right\} \cup\{x\}$ and define a map $\tilde{h}: M \times G \rightarrow M \times \widehat{G}$ by

$$
\tilde{h}\left(x_{n}, s\right):=\left(x_{n}, h_{\omega_{x_{n}} \circ \sigma_{n}}(s)\right) \quad \text { and } \quad \tilde{h}(x, s)=h^{\alpha}(x, s)
$$

for all $n \in \mathbb{N}$ and $s \in G$, where for any 2-cocycle $\omega$ on $G$ and $\sigma \in \operatorname{Aut}(G)$, $\omega \circ \sigma(s, t)=\omega(\sigma(s), \sigma(t))$. Since $h^{\alpha}$ is continuous by [6. Lemma 5.5.4] and $\sigma_{n} \rightarrow \mathrm{id}_{G}$ it follows that $\tilde{h}$ is continuous, too, and we proceed by showing that it is open as a map onto its image. For this we put $\tilde{h}_{x_{n}}=h_{\omega_{x_{n}} \circ \sigma_{n}}$ for all $n \in \mathbb{N}$ and $\tilde{h}_{x}=h_{\omega_{x}}$. Furthermore, for $n \in \mathbb{N}$, let $\tilde{\Sigma}_{x_{n}}=\sigma_{n}^{-1}\left(\Sigma_{x_{n}}\right)=\operatorname{ker} \tilde{h}_{x_{n}}$, and let $\tilde{\Sigma}_{x}=\Sigma_{x}=\operatorname{ker} \tilde{h}_{x}$. Then $\tilde{\Sigma}_{x_{n}} \cap V=\Sigma_{x} \cap V$ for all $n \in \mathbb{N}$, and we may pass over to $G /\left(\Sigma_{x} \cap V\right)$ in order to show the openness of $\tilde{h}$. After having done this, we may assume that $\tilde{\Sigma}_{x_{n}}$ has trivial intersection with the connected component $G_{0}$ of $G$ for all $n \in \mathbb{N}$, and the same may be assumed for $\tilde{\Sigma}_{x}$. Moreover, if $q: G \rightarrow G / G_{0}$ denotes the quotient map, then it follows from our constructions that $q\left(\tilde{\Sigma}_{x_{n}}\right)=q\left(\tilde{\Sigma}_{x}\right)$ for all $n \in \mathbb{N}$. 
Let $H=\tilde{\Sigma}_{x} \cdot G_{0}$. Then also $H=\tilde{\Sigma}_{y} \cdot G_{0}$ for all $y \in M\left(\right.$ since $\left.q\left(\tilde{\Sigma}_{x_{n}}\right)=q\left(\tilde{\Sigma}_{x}\right)\right)$, and we conclude that

$$
\tilde{h}_{y}(s H)=\tilde{h}_{y}(s) \tilde{h}_{y}\left(\tilde{\Sigma}_{y} \cdot G_{0}\right)=\tilde{h}_{y}(s) \tilde{h}_{y}\left(G_{0}\right)
$$

lies in the connected component of $\widehat{G}$ if and only if $s \in H$. Suppose now that $\left(s_{n}\right)_{n \in \mathbb{N}}$ is a sequence in $G$ such that $\tilde{h}\left(x_{n}, s_{n}\right) \rightarrow \tilde{h}(x, s)$ for some $s \in G$. Multiplying each $s_{n}$ with $s^{-1}$ we may assume that $\tilde{h}\left(x_{n}, s_{n}\right) \rightarrow\left(x, 1_{G}\right)$ in $M \times \widehat{G}$. Since $M \times(\widehat{G})_{0}$ is open in $M \times \widehat{G}$, it follows from the above observation that $s_{n} \in H$ for all but finitely many $n \in \mathbb{N}$. Thus, by multiplying this sequence with $z_{n}$ in the second variable, for appropriate elements $z_{n} \in \tilde{\Sigma}_{x_{n}}$, we may assume that $s_{n} \in G_{0}$ for all $n \in \mathbb{N}$ and that $\tilde{h}\left(x_{n}, s_{n}\right) \rightarrow\left(x, 1_{G}\right)$ in $M \times \widehat{G}$. We want to show that $s_{n} \rightarrow e$ in $G$. For this we write $G_{0}=W \times T$ for a vector group $W$ and a torus group $T$, and we write $s_{n}=r_{n} \cdot t_{n}, r_{n} \in W$ and $t_{n} \in T$. By the compactness of $T$ we may assume that $t_{n} \rightarrow t$ for some $t \in T$. It follows that $s_{n} t_{n}^{-1} \in W$ for all $n \in \mathbb{N}$, and that

$$
\tilde{h}_{x_{n}}\left(s_{n} t_{n}^{-1}\right)=\tilde{h}_{x_{n}}\left(s_{n}\right) \tilde{h}_{x_{n}}\left(t_{n}^{-1}\right) \rightarrow \tilde{h}_{x}\left(t^{-1}\right) .
$$

Since $\tilde{h}_{x}$ maps the torus part of $G$ into the torus part of $\widehat{G}$, it follows that $\left.\tilde{h}_{x}\left(t^{-1}\right)\right|_{W}$ $=1_{W} \in \widehat{W}$. Thus $\left.\tilde{h}_{x_{n}}\left(s_{n} t_{n}^{-1}\right)\right|_{W} \rightarrow 1_{W}$ in $\widehat{W}$. Since each $\tilde{\Sigma}_{x_{n}}$ has trivial intersection with $G_{0}$, and hence also with $W$, we see that the restriction of $\omega_{x_{n}} \circ \sigma_{n}$ to $W \times W$ is totally skew for all $n \in \mathbb{N}$, and the same applies to the restriction of $\omega_{x}$ to $W \times W$. Thus, for each $y \in M$, we obtain an isomorphism $W \rightarrow \widehat{W}$ given by $\left.s \mapsto \tilde{h}_{y}(s)\right|_{W}$. By identifying $W$ with $\widehat{W}$ via any fixed linear isomorphism, the continuity of $\tilde{h}$ implies that the map $M \rightarrow \operatorname{GL}(W) ;\left.y \mapsto \tilde{h}_{y}\right|_{W}$ is continuous, too. Thus, the map $M \rightarrow \mathrm{GL}(W) ; y \mapsto\left(\left.\tilde{h}_{y}\right|_{W}\right)^{-1}$ is also continuous. This implies that $s_{n} t_{n}^{-1} \rightarrow\{0\}$ in $W$, which in turn yields $s_{n} \rightarrow t$ in $G$. But, since by assumption $\tilde{h}_{x_{n}}\left(s_{n}\right) \rightarrow \tilde{h}_{x}(t)=1_{G}$ in $\widehat{G}$, and since $\operatorname{ker} \tilde{h}_{x} \cap G_{0}=\Sigma_{x} \cap G_{0}=\{e\}$, it follows that $t=1$. This completes the proof for the openness of $\tilde{h}$.

Finally, let $\left(s_{n}\right)_{n \in \mathbb{N}}$ be a sequence in $G$ such that $h^{\alpha}\left(x_{n}, s_{n}\right) \rightarrow h^{\alpha}(x, s)$ for some $s \in G$. Then it follows that $\tilde{h}\left(x_{n}, \sigma_{n}^{-1}\left(s_{n}\right)\right)=\left(x_{n}, h_{\omega_{x_{n}}}\left(s_{n}\right) \circ \sigma_{n}\right)$ converges to $\left(x, h_{\omega_{x}}(s)\right)=\tilde{h}(x, s)$ in $M \times \widehat{G}$. Since $\tilde{h}$ is open as a map onto its image, we may multiply each $\sigma_{n}\left(s_{n}\right)$ with an appropriate element $r_{n} \in \tilde{\Sigma}_{x_{n}}=\sigma_{n}^{-1}\left(\Sigma_{x_{n}}\right)$ such that, after possibly passing to a subsequence, $\left(x_{n}, \sigma_{n}^{-1}\left(s_{n}\right) r_{n}\right) \rightarrow(x, s)$ in $M \times G$. Thus, if $r_{n}^{\prime}=\sigma_{n}\left(r_{n}\right)$, we have $r_{n}^{\prime} \in \Sigma_{x_{n}}$ for all $n \in \mathbb{N}$ and $\left(x_{n}, s_{n} r_{n}^{\prime}\right) \rightarrow(x, s)$ in $M \times G \subseteq \Omega \times G$. This completes the proof.

\section{ACKNOWLEDGEMENTS}

Much of this work was done while the first author visited Dartmouth College in January 2005. He wants to take this opportunity to thank the members of the Department of Mathematics, and especially Professor Dana Williams, for their very warm hospitality.

\section{REFERENCES}

[1] William Arveson, An Invitation to $C^{*}$-algebras, Springer-Verlag, New York, 1976. Graduate Texts in Mathematics, No. 39. MR0512360 (58:23621)

[2] Larry Baggett and Adam Kleppner, Multiplier representations of abelian groups, J. Functional Analysis 14 (1973), 299-324. MR 51:791

[3] Teresa Bates, David Pask, Iain Raeburn, and Wojciech Szymański, The $C^{*}$-algebras of rowfinite graphs, New York J. Math. 6 (2000), 307-324 (electronic). MR1777234(2001k:46084) 
[4] Klaus Deicke, Pointwise unitary coactions on $C^{*}$-algebras with continuous trace, J. Operator Theory 43 (2000), no. 2, 295-327. MR2001d:46098

[5] Jacques Dixmier, von Neumann algebras, North-Holland Mathematical Library, vol. 27, North-Holland Publishing Co., Amsterdam, 1981. With a preface by E. C. Lance, Translated from the second French edition by F. Jellett. MR641217(83a:46004)

[6] Siegfried Echterhoff, Crossed products with continuous trace, Mem. Amer. Math. Soc. 123 (1996), no. 586, i-viii, 1-134. MR.98f:46055

[7] Edward G. Effros, A decomposition theory for representations of $C^{*}$-algebras, Trans. Amer. Math. Soc. 107 (1963), 83-106. MR26:4202

[8] Gerald B. Folland, A course in abstract harmonic analysis, Studies in Advanced Mathematics, CRC Press, Boca Raton, FL, 1995. MR1397028 (98c:43001)

[9] James Glimm, Families of induced representations, Pacific J. Math. 12 (1962), 885-911. MR0146297 (26:3819)

[10] Elliot C. Gootman and Jonathan Rosenberg, The structure of crossed product $C^{*}$-algebras: A proof of the generalized Effros-Hahn conjecture, Invent. Math. 52 (1979), no. 3, 283-298. MR 80h:46091

[11] Philip Green, The local structure of twisted covariance algebras, Acta Math. 140 (1978), 191-250. MR0493349 (58:12376)

[12] Alex Kumjian, David Pask, Iain Raeburn, and Jean Renault, Graphs, groupoids, and CuntzKrieger algebras, J. Funct. Anal. 144 (1997), no. 2, 505-541. MR1432596 (98g:46083)

[13] Alex Kumjian and David Pask, $C^{*}$-algebras of directed graphs and group actions, Ergodic Theory Dynam. Systems 19 (1999), no. 6, 1503-1519. MR.1738948 (2000m:46125)

[14] Ronald Lipsman, Representation theory of almost connected groups, Pacific J. Math. 42 (1972), 453-467. MR0327975 (48:6317)

[15] George W. Mackey, Imprimitivity for representations of locally compact groups. I, Proc. Nat. Acad. Sci. U. S. A. 35 (1949), 537-545. MR0031489(11:158b)

[16] Calvin C. Moore and Jonathan Rosenberg, Groups with $T_{1}$ primitive ideal spaces, J. Functional Analysis 22 (1976), no. 3, 204-224. MR0419675 (54:7693)

[17] Dorte Olesen and Iain Raeburn, Pointwise unitary automorphism groups, J. Funct. Anal. 93 (1990), no. 2, 278-309. MR92b:46105

[18] Detlef Poguntke, Der Raum der primitiven Ideale von endlichen Erweiterungen lokalkompakter Gruppen, Archiv Math. 28 (1977), 133-138. MR0439981(55:12862)

[19] Iain Raeburn and Dana P. Williams, Morita equivalence and continuous-trace $C^{*}$-algebras, Mathematical Surveys and Monographs, vol. 60, American Mathematical Society, Providence, RI, 1998. MR2000c:46108

[20] Jean-Luc Sauvageot, Idéaux primitifs de certains produits croisés, Math. Ann. 231 (1977/78), no. 1, 61-76. MR473355 (80d:46112)

[21] _ Idéaux primitifs induits dans les produits croisés, J. Funct. Anal. 32 (1979), no. 3, 381-392. MR81a:46080

[22] Masamichi Takesaki, Covariant representations of $C^{*}$-algebras and their locally compact automorphism groups, Acta Math. 119 (1967), 273-303.

[23] Nik Weaver, A prime $C^{*}$-algebra that is not primitive, J. Funct. Anal. 203 (2003), no. 2, 356-361. MR2003352 (2004g:46075)

[24] Dana P. Williams, The topology on the primitive ideal space of transformation group $C^{*}$ algebras and C.C.R. transformation group $C^{*}$-algebras, Trans. Amer. Math. Soc. 266 (1981), no. 2, 335-359. MR617538 (82h:46081)

[25] Georges Zeller-Meier, Produits croisés d'une $C^{*}$-algèbre par un groupe d'automorphismes, J. Math. Pures Appl. (9) 47 (1968), 101-239. MR0241994 (39:3329)

Mathematisches Institut, Westfälische Wilhelms-Universität Münster, Einsteinstr. 62, W-48149 Münster, Germany

E-mail address: echters@math.uni-muenster.de

Department of Mathematics, Dartmouth College, Hanover, New Hampshire 037553551

E-mail address: dana.williams@dartmouth.edu 